\title{
COMMENT
}

Check for updates

\section{Sustainable social development: tackling poverty to achieve kidney health equity}

\section{R. Moosa ${ }^{1}$ and K. C. Norris $\mathbb{1}^{2 凶}$}

Chronic kidney disease (CKD) is a rapidly growing public health problem, especially in disadvantaged populations. Major political interventions are required to mitigate the social and socioeconomic inequities that contribute to the development and progression of CKD and its disproportionate impact on low and middle-income countries.
In communities living in poverty, the risk of developing CKD begins to accrue even before birth
${ }^{1}$ Department of Medicine Faculty of Medicine and Health Sciences, Stellenbosch University, Cape Town, South Africa.

${ }^{2}$ Department of Medicine, UCLA School of Medicine, Los Angeles, CA, USA.

凶e-mail: knorris@ucla.edu https://doi.org/10.1038/ s41581-020-00342-x
The world is coming to terms with the ravages wrought by the novel coronavirus SARS-CoV-2, which causes coronavirus disease 2019 (COVID-19). At the time of writing, the virus has already caused more than 650,000 deaths worldwide and is predicted to plunge the globe into an economic recession that will rival that of the Great Depression. However, another silent epidemic chronic kidney disease (CKD) - claimed more than 7.1 million lives in 2010 alone and is estimated to claim 9.1 million lives in 2030. This disease takes a heavy toll at the population level and places a major burden on the economies of countries. The majority of patients with CKD live in low and middle-income countries (LMICs) where kidney failure devastates households, pushing many families into extreme poverty ${ }^{1}$. Access to kidney replacement therapy (KRT) is strikingly unequal - fewer than $4 \%$ of patients living in LICs have access to treatment compared with $60 \%$ of those living in high-income countries (HICs). Moreover, although the incidence of CKD has plateaued in HICs, it continues to increase substantially in LMICs. This stabilization in HICs has been ascribed to successful CKD prevention and/or slowing of its progression to kidney failure ${ }^{2}$. The burden of diabetes is increasing at a faster rate in LMICs than in HICs, which, in combination with ageing of the population, the rise in obesity and the burden of infectious diseases, further contributes to the rising incidence of CKD in $\mathrm{LMICs}^{3}$. Ultimately, much of this increase in incidence is related directly or indirectly to the consequences of poverty. Therefore, progress towards achieving the 17 Sustainable Development Goals adopted in 2015 has the potential to contribute to the prevention and management of $\mathrm{CKD}^{4}$.

In communities living in poverty, the risk of developing CKD begins to accrue even before birth and prevails throughout the life of the individual (Supplementary Box 1). Moreover, poverty is a risk factor not only for developing CKD but also for progression towards kidney failure. The complex interplay between biological factors (such as age, hypertension and diabetes) and social determinants of health (such as socioeconomic factors, psychosocial issues, the environment and access to health care) are postulated to be responsible for this inequity (Supplementary Box 1$)^{5}$. Importantly, within a country, the degree of income disparity (measured by the Gini coefficient) contributes to gross inequities between wealthy and poor individuals, and leads to disparate health care even in HICs - a HIC with a high Gini coefficient might offer poorer health care to its citizens than an LMIC with a low Gini coefficient ${ }^{6}$.

What needs to change? Many of the solutions for the prevention and care of CKD must work around a global structure of inequity, and these imbalances are magnified in LIMCs, which carry an excess burden of premature morbidity and mortality. The ultimate solution lies in addressing these inequities but this is no small task. The WHO has outlined three principles of global action to address injustices that influence the social determinants of health, with the aim of achieving health equity within a generation - a bold goal ${ }^{7}$. First, living and working conditions must improve for all to ensure that, from birth to older age, all individuals can achieve their full health potential. Second, societies must be organized to ensure that power, money and resources are distributed equitably. Third, progress depends on methodical assessment of the problems and judicious evaluation of solutions, and efforts must therefore be made to educate, not only health-care workers but also the wider society, on how social inequity drives health disparities.

Investing in primary prevention is an obvious approach to addressing the global CKD problem but, in reality, the insidiousness of CKD onset limits this strategy, especially in LMICs, where the majority of patients present late in the course of disease, and CKD is typically only detected when the patient is already experiencing kidney failure. Nonetheless, improving 


Many of the
solutions for
the prevention
and care of
CKD must work
around a global
structure of
inequity

the care of patients with diabetes and hypertension is a time-honoured approach to the prevention of CKD that should considerably reduce the number of patients who need the attention of a nephrologist. Indeed, for many LMICs, primary prevention might include developing a culture of health, including promoting and facilitating access to healthy foods, investing in education about CKD and its risk factors, as well as improving living conditions by safeguarding environmental justice and ensuring equitable access to housing and employment ${ }^{3,7}$.

Beyond disease prevention, improving disease management is of crucial importance. The WHO should be encouraged to continue to advocate for the attenuation of the social drivers of non-communicable diseases in LMICs, such as wealth inequity and discrimination, and to highlight the value of coordinated multisectoral interventions, which are often supported by nongovernmental organizations. Examples of such initiatives include improved urban planning with sanitation control and access to clean water, and the elimination of environmental toxins, for example, by controlling the use of agricultural pesticides and industrial chemicals ${ }^{3}$. Many governmental agencies themselves represent substantial barriers to improving the management of $\mathrm{CKD}$, including the rollout of KRT, because they are not aware of the devastating impact of kidney disease. Therefore, the advocacy efforts of international organizations such as the International Society of Nephrology (ISN) must supplement those of local and/or regional organizations - these efforts are crucial and must be harnessed to persuade national governments of the need for action ${ }^{8}$. Furthermore, HICs can assist by offering to train staff from LMICs, either directly or as part of global initiatives such as the very successful ISN Fellowships. These are some of the 'political' strategies that might assist in mitigating the inequities alluded to earlier. The current global initiative towards universal health coverage driven by the WHO, United Nations and World Bank must also be supported as an important approach to improving health-care access and mitigating socioeconomic inequities.

From an economic viewpoint, countries can improve cost efficiencies by buying in bulk, using mid-level workers and using cheaper forms of dialysis. Decentralized forms of treatment, including home haemodialysis, are more patient-centred and technically less costly than in-centre haemodialysis, but many barriers might affect the feasibility of this approach in LMICs, ranging from inadequate home plumbing to support the water requirements of dialysis, to insufficient health policy coverage ${ }^{9}$. Thus, although home dialysis should be seriously considered, especially in the wake of the COVID-19 pandemic when physical distancing is a key component of reducing exposure to the virus, in many LMICs home treatment will be limited to peritoneal dialysis. In addition, innovative technologies and strategies must be sought that will reduce the overall cost of dialysis but allow safe and effective treatment even in poor communities.

The three principles of global action outlined by the WHO can help create a foundation for health equity and, in turn, reduce avoidable risk factors for CKD and liberate resources that can be redirected towards $\mathrm{CKD}$ prevention, early intervention and, where necessary, cost-effective KRT. COVID-19 has placed a spotlight on these inequities as society's most vulnerable people are yet again dying at disproportionately high rates. Of note, most of the clinical risk factors for COVID-19-related death are also risk factors for CKD ${ }^{10}$. Therefore, the COVID-19 pandemic has unmasked the insidious pandemic of non-communicable diseases such as CKD. Like chronic and acute communicable diseases, non-communicable diseases might not discriminate, but society does, and the repercussions resonate strongly in LMICs. Remaining steadfast in a concerted effort to address the social determinants of health, while simultaneously advancing education about CKD and CKD risk factors, improving screening and delivering early intervention remain paramount in our quest to improve the health of all citizens, their families and their communities.

1. International Society of Nephrology. Global Health Atlas 2019. https://www theisn org/focus/ckd\#health-atlas (ISN, 2019).

2. Robinson, B. M. et al. Factors affecting outcomes in patients reaching end-stage kidney disease worldwide: differences in access to renal replacement therapy, modality use, and haemodialysis practices. Lancet 388, 294-306 (2016).

3. Stanifer, J. W., Muiru, A., Jafar, T. H. \& Patel, U. D. Chronic kidney disease in low- and middle-income countries. Nephrol. Dial. Transplant. 31, 868-874 (2016).

4. Luyckx, V. A., Tonelli, M. \& Stanifer, J. W. The global burden of kidney disease and the sustainable development goals. Bull World Health Organ. 96, 414-422D (2018).

5. Norton, J. M. et al. Social determinants of racial disparities in CKD. J. Am. Soc. Nephrol. 27, 2576-2595 (2016).

6. Sen, A. Development as Freedom (Knopf, 1999)

7. World Health Organization. Closing the Gap in a Generation Health Equity Through Action on the Social Determinants of Health (WHO, 2008).

8. Bello, A. K. et al. Complications of chronic kidney disease: current state, knowledge gaps, and strategy for action. Kidney Int. Suppl. 7 122-129 (2017).

9. Walker, R. C. Howard, K. \& Morton, R. L. Home hemodialysis: a comprehensive review of patient-centered and economic considerations. Clinicoecon. Outcomes Res. 9, 149-161 (2017).

10. Zhou, F. et al. Clinical course and risk factors for mortality of adult inpatients with COVID-19 in Wuhan, China: a retrospective cohort study. Lancet 395, 1054-1062 (2020).

\section{Acknowledgements}

K.C.N. is supported by NIH grants P30AG021684 and UL1TR001881.

\section{Competing interests}

The authors declare no competing interests.

Supplementary information

Supplementary information is available for this paper at https://doi.org/ 10.1038/s41581-020-00342-x. 